\title{
Modern system approach to the diagnosis, therapy and rehabilitation of mental disorders
}

Mitikhin V., Solokhina T., Tiumenkova G. Mental Health Research Centre, Moscow, Russian Federations

Introduction The current stage of research on mental disorders is associated with the use of system approaches to the development of the scientific foundations of psychiatric care.

Modern approaches to solving the main problems in psychiatry related to the classification, diagnosis, treatment and rehabilitation of mental disorders are based on the integration of clinical, psychometric, social and neurobiological characteristics of patients. These characteristics correspond to the measurement scales: categorical, rank (dimensional, psychometric) and the scale of relations. Rank estimates do not allow calculating even average values [1], much less creating correct models of states taking into account neurobiological (numerical) indicators.

Objectives Approach to solving problems that arise in the diagnosis of psychopathological conditions, assessing their severity, as well as evaluating the effectiveness of psychosocial treatment and rehabilitation.

Methods Clinical, psychometric, system analysis methods and algorithms of the Analytical Hierarchy Process (AHP) [1] were used.
Results When assessing a patient's condition and behavior, it is necessary to make decisions (diagnosis, development of treatment and rehabilitation plans) based on heterogeneous information (genetic, neuronal and environmental, involving individual characteristics, as well as family and social context). This information is hierarchically organized and includes quantitative and qualitative data.

Exposure at each of these different levels can affect the onset and course of the disease, and therefore should be considered in primary prevention and subsequent psychosocial therapy and rehabilitation of patients.

Analysis of the problems of assessing psychopathological states and related psychosocial problems shows that these problems can be presented in the form of appropriate hierarchies, the structure of which must be taken into account when processing the initial information. As an example, we give a hierarchy for the integral assessment of patient compliance.

As an example, we give a hierarchy for the integral assessment of patient compliance.
Where: 1) S1-attitude to therapy (13 factors: F1-F13); 2) S2-factors related to the patient (8 factors: F14-F21); 3) S3 - factors related to the immediate environment ( 2 factors: F22-F23); 4) S4 - factors related to the attending physician (2 factors: F24-F25).

Note that the levels of these factors F1-F 25 are operationally set in rank scales, so to determine their weights, we should use the normative approach of AHP (the algorithms of this approach are described, for example, in [1]). To determine the weights of the S1S4 subscales, whose preferences are evaluated only on the basis of expert information, the descriptive approach of AHP should be used [1].

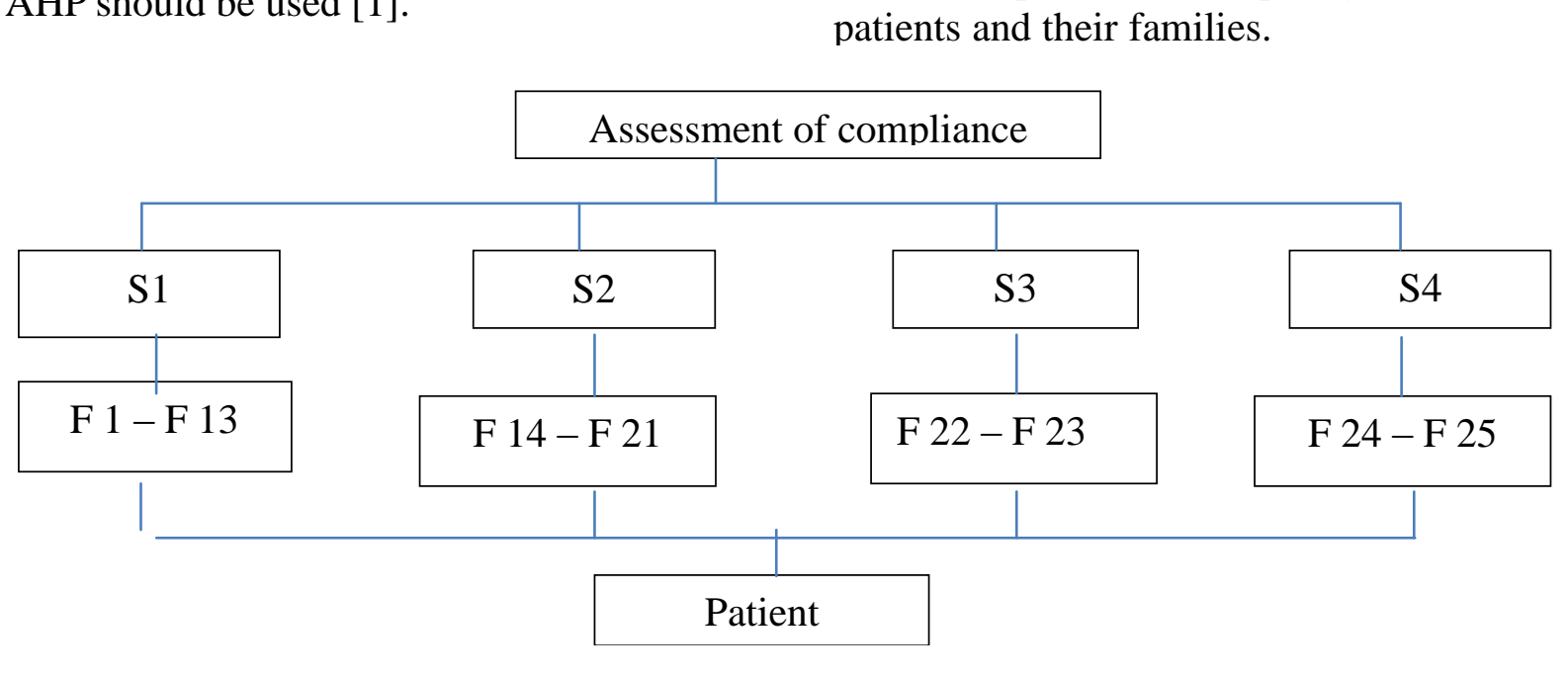

Conclusions The main advantages of the AHP include the use of the relationship scale (fundamental scale) for processing heterogeneous data based on expert, clinica information.

The developed models for assessing the level of patient compliance and identifying patient characteristics that are significant for compliance, characteristics of the family environment and factors of psychiatric care, allow us to increase the effectiveness of therapy, the choice of personal strategies fo psychosocial and psychoeducational interventions, reduce the risk of repeated hospitalizations and increase the level of social adaptation and quality of life of patients and their families.

.

